\title{
Propuesta de políticas sobre cuidados paliativos para personas adultas mayores en Latinoamérica y el Caribe
}

Jose F. Parodi ${ }^{1}$, Rocío Morante ${ }^{2}$, Lilian Hidalgo ${ }^{3}$, Ricardo Carreño ${ }^{4}$

RESUMEN

El rápido envejecimiento poblacional, los cambios epidemiológicos y la necesidad de garantizar derechos humanos (salud, protección social, etc.), obligan a que los estados y la comunidad en general tome conciencia y desarrolle políticas que sirvan de base para una estrategia de mantenimiento de la salud y cuidado de los adultos mayores. Esto debe diseñarse tomando en cuenta las particularidades de este grupo etáreo, y la necesidad de adecuación de los servicios socio sanitario para brindar un cuidado con calidad y equidad.

La adecuación de los servicios implica el reconocimiento las nuevas necesidades, las particularidades de los riesgos y problemas de esta etapa de vida, la atención integral, e inclusive el cuidado digno y basado en evidencia al final de la vida. Ante nuevos problemas y nuevas metas, se requieren nuevas competencias, nuevos escenarios de trabajo y un sistema de información que permita mejorar la eficiencia y calidad de las intervenciones.

Palabras clave: Cuidados paliativos, anciano, adulto mayor, políticas de salud, envejecimiento.

\section{Proposed policies on palliative care for elderly people in Latin America and the Caribbean}

\section{ABSTRACT}

The fast aging of population, epidemiological changes and the need to guarantee human rights (health, social protection, etc.) force countries and the wider community to become aware and develop policies that form the basis for an strategy of maintaining the health and care of elderly people. This should be designed taking into account the particularities of this age group, and the need for adaptation of social health services to provide quality care and equity. The adequacy of services involves recognizing the new requirements, the particularities of the risks and problems of this stage of life, comprehensive care, and even decent evidence-based on the end of life.

In the presence of new problems and new goals, new skills, new work scenarios and an information system that improve the efficiency and quality of interventions are required

Keywords: Palliative care; elderly; older adult; health policy; aging (MeSH Terms)

\footnotetext{
${ }^{1}$ Médico Geriatra. Centro de Investigación del Envejecimiento del Instituto de Envejecimiento. Facultad de Medicina Humana. Universidad San Martín de Porres. Magister en Salud Pública y Epidemiología.

2 Médico Cirujano de Bamboo Senior Health Services. Graduada del Curso en Gerencia de Salud para Personas Mayores OPS/ALMA/CIESS.

3 Médico Geriatra con acreditación en Cuidados Paliativos. OncoSalud de Lima.

${ }_{4}^{4}$ Médico Epidemiólogo. Instituto Nacional de Enfermedades Neoplásicas. Perú. Magister el Gobierno y Gerencia en Salud.
} 


\section{INTRODUCCIÓN}

El cambio demográfico y epidemiológico, de la mano del avance de la medicina, ha condicionado que se presenten nuevos usuarios y nuevos problemas para la organización de los servicios de salud. La mejora del acceso a servicios adecuados a las particularidades de los nuevos usuarios es una obligación de los sistemas de salud. El crecimiento de la carga de enfermedad crónica no transmisible, incluyendo el cáncer y las enfermedades degenerativas, ha impulsado el desarrollo de una rama de la medicina llamada Cuidados Paliativos $(C P)$. Frente a este aumento progresivo de las necesidades de cuidados paliativos, la capacidad de respuesta en América Latina y el Caribe (ALC) es escasa: muchos países no satisfacen las demandas y se considera que sólo reciben cuidados paliativos entre el 5 y el $10 \%$ de los enfermos que los necesitan (1); más del $90 \%$ de servicios paliativos está en las grandes ciudades, y más del $50 \%$ de pacientes no puede pagar los servicios o la medicación. El $80 \%$ de las naciones no los reconocen como una disciplina y no los incluyen en los sistemas sanitarios públicos o privados (2).

El desarrollo de los CP en ALC ha sido errático y sin un patrón definido, por lo que están en distintas etapas de desarrollo. Siguiendo la clasificación de Wright y colaboradores, 11 países están en prestación aislada de servicios, 6 en etapa de integración preliminar con los servicios de salud estándar y 2 países (Colombia y Costa Rica) se consideran en una etapa de integración avanzada con los servicios de salud estándar. Pese a los esfuerzos, la mayoría de la población sigue sin cobertura de CP(3).

\section{Un escenario demográfico y epidemiológico diferente}

En la actualidad, $60 \%$ de las personas mayores (PAM) de 60 años viven en ALC, y se espera que para el año 2025 este porcentaje ascienda a 75\%. A modo de ejemplo, México pasará de tener una población mayor de 60 años cercana al 7\% en el año 2000, a casi $15 \%$ en 2025 , mientras que los adultos mayores pasarán en Brasil de ser el $8 \%$ a algo más de $15 \%$ en ese mismo lapso (4) .

En los países en desarrollo se está produciendo adicionalmente -donde no se ha producido ya- el envejecimiento de los adultos mayores, de modo que la esperanza de vida a los 60 años para un ciudadano en esos países es similar a la observada en los países más industrializados (5). Así, la esperanza de vida de una mujer canadiense a los 60 años de edad era de 24 años en 1996, mientras que la de una mexicana ya era de 23 años (6).

En el campo de la salud, las consecuencias de este envejecimiento poblacional son especialmente marcadas. Esto se debe a la concurrencia, junto a la transición demográfica, de una transición epidemiológica que ha llevado a que las enfermedades infecciosas sean sustituidas por las no infecciosas, habitualmente crónicas, muy ligadas a determinados estilos de vida y que se presentan preferentemente a edades avanzadas (7). Pero esta transformación demográfica y epidemiológica que se ha producido nítidamente en los países industrializados, ofrece perfiles más confusos en los países en vías de desarrollo. Es posible que en América Latina las enfermedades transmisibles tradicionales sigan existiendo junto con las no transmisibles oncológicas y no oncológicas durante varias décadas, por lo que interactúan, entre sí y presentan alto potencial de llegar a la discapacidad y terminalidad (7). Esta situación ha originado lo que algunos autores denominan la "doble carga de la enfermedad" sobre los sistemas sanitarios de estos países(8).

Todo esto constituye un escenario diferente ante el cual los estados, los servicios socio sanitario y la comunidad misma deben estar preparados.

La salud de las personas con enfermedades en estado terminal en la política pública y su adaptación a los instrumentos internacionales de derechos humanos

El logro del máximo grado de bienestar de la población - incluidos los ciudadanos con enfermedad en estado terminal - exige que se compartan entre el estado y los ciudadanos tanto el compromiso como la responsabilidad. Los Estados deben crear las condiciones propicias para formular políticas y marcos normativos para su ejecución, en respuesta a los objetivos del milenio en sus respectivos países. Es importante resaltar que la implementación de servicios de CP es factible y costo-efectiva sobre todo si se realiza en colaboración con países con problemática y sistemas sanitarios similares, y con la ayuda de organizaciones expertas (2).

Al respecto existen tres puntos fundamentales que deberían guiar la implementación del marco político en salud de las PAM, incluidos aquellos con enfermedades terminales: 
1. Formular políticas, leyes, reglamentos, programas y presupuestos en cuidados paliativos congruentes con los instrumentos sobre derechos humanos del sistema de las Naciones Unidas y del sistema interamericano (OEA). Según la publicación de 2012, solo tres países de la región tienen una ley de Cuidados Paliativos. Hay planes/programas nacionales de Cuidados
Paliativos en 7 países, 5 de ellos integrados con cáncer y dolor. (Tabla 1 ).

Por lo tanto, la meta sería que en un plazo no mayor de diez años todos los países de la región cuenten con una política, un marco legal y un plan nacional de cuidados paliativos.

Tabla 1. Situación de los Cuidados Paliativos según países en América Latina

\begin{tabular}{|c|c|c|c|c|c|c|c|c|c|c|c|c|}
\hline \multirow{2}{*}{$\begin{array}{c}\text { País } \\
\text { Argentina }\end{array}$} & \multirow{2}{*}{$\begin{array}{c}\text { Ley } \\
\text { nacional } \\
\text { No* }^{*}\end{array}$} & \multirow{2}{*}{$\begin{array}{c}\text { Programa/ } \\
\text { Plan } \\
\text { nacional } \\
\text { No }\end{array}$} & \multirow{2}{*}{$\begin{array}{c}\text { Auditoria, } \\
\text { monitoreo, } \\
\text { evaluación } \\
\text { No }\end{array}$} & \multicolumn{2}{|c|}{$\begin{array}{c}\text { Programa } \\
\text { Nacional } \\
\text { de } \\
\text { Cáncer } \\
\text { (incluye } \\
\text { CP) }\end{array}$} & \multicolumn{2}{|c|}{$\begin{array}{c}\text { Programa } \\
\text { Nacional } \\
\text { de VIH/ } \\
\text { SIDA } \\
\text { (incluye } \\
\text { CP) }\end{array}$} & \multicolumn{2}{|c|}{$\begin{array}{c}\text { Programa } \\
\text { Nacional } \\
\quad \text { de } \\
\text { Atención } \\
\text { Primaria } \\
\text { (incluye } \\
\text { CP) }\end{array}$} & \multirow{2}{*}{$\begin{array}{c}\text { Recursos } \\
\text { para } \\
\text { desarrollo } \\
\text { No }\end{array}$} & \multirow{2}{*}{$\begin{array}{c}\text { Recursos } \\
\text { para } \\
\text { investigación }\end{array}$} & \multirow{2}{*}{$\begin{array}{c}\text { Opioides: } \\
\text { Colaboración } \\
\text { entre } \\
\text { prescriptores } \\
y \\
\text { reguladores } \\
4.0\end{array}$} \\
\hline & & & & $\mathrm{Si}$ & (Si) & $\mathrm{Si}$ & (Si) & $\mathrm{Si}$ & (No) & & & \\
\hline Bolivia & No & No & No & No & --- & $\mathrm{Si}$ & (No) & $\mathrm{Si}$ & (No) & No & No & 1.0 \\
\hline Brasil & No & $\mathrm{Si+}$ & No & $\mathrm{Si}$ & (Si) & $\mathrm{Si}$ & (Si) & $\mathrm{Si}$ & $(\mathrm{Si})$ & No & No & 3.5 \\
\hline Chile & $\mathrm{Si}$ & $\mathrm{Si+}$ & Si & $\mathrm{Si}$ & (Si) & $\mathrm{Si}$ & (Si) & $\mathrm{Si}$ & (Si) & $\mathrm{Si}$ & No & 4.0 \\
\hline Colombia & $\mathrm{Si+}$ & No & No & $\mathrm{Si}$ & (Si) & $\mathrm{Si}$ & (No) & $\mathrm{Si}$ & (No) & No & $\mathrm{Si}$ & 3.5 \\
\hline Costa Rica & No & No & Si & $\mathrm{Si}$ & (Si) & $\mathrm{Si}$ & (Si) & $\mathrm{Si}$ & $(\mathrm{Si})$ & $\mathrm{Si}$ & No & 5.0 \\
\hline Cuba & No & $\mathrm{Si+}$ & $\mathrm{Si}$ & $\mathrm{Si}$ & $(\mathrm{Si})$ & $\mathrm{Si}$ & $(\mathrm{Si})$ & $\mathrm{Si}$ & (Si) & $\mathrm{Si}$ & $\mathrm{Si}$ & 4.0 \\
\hline Ecuador & No & No & No & $\mathrm{Si}$ & (Si) & $\mathrm{Si}$ & (No) & $\mathrm{Si}$ & (No) & No & No & 3.0 \\
\hline EI Salvador & No & No & No & $\mathrm{Si}$ & (No) & $\mathrm{Si}$ & (No) & $\mathrm{Si}$ & (No) & No & No & 2.0 \\
\hline Guatemala & No & No & No & No & --- & Si & (No) & $\mathrm{Si}$ & (No) & No & No & 2.0 \\
\hline Honduras & No & No & No & $\mathrm{Si}$ & (No) & $\mathrm{Si}$ & (No) & $\mathrm{Si}$ & (No) & No & No & 1.3 \\
\hline México & $\mathrm{Si}$ & $\mathrm{Si}$ & No & $\mathrm{Si}$ & (Si) & $\mathrm{Si}$ & (Si) & $\mathrm{Si}$ & (No) & No & $\mathrm{Si}$ & 3.0 \\
\hline
\end{tabular}

*Hay leyes federales, estatales o municipales. +Vinculado a programas de cáncer o dolor. † Recursos del Instituto Nacional del Cáncer

2. Formular marcos legales para los CP y sus mecanismos de ejecución en personas mayores que están en servicios de cuidados de largo plazo. Desde luego, esto implica asignar el mecanismo, presupuesto y el personal adecuado para las actividades de supervisión intersectorial.

La meta sería que en un plazo determinado, todos los países de la región garanticen el acceso a CP para las personas en los servicios de cuidados de largo plazo.

3. Fomentar la cooperación de los países y entre ellos en cuanto al diseño de estrategias y el intercambio de capacidades y recursos para la construcción y desarrollo de sus marcos políticos en relación a CP.
Consecuentemente, la meta sería que los países de la región, en un plazo determinado, deben contar al menos con una alianza destinada a ejecutar sus planes nacionales sobre CP.

4. Fomentar servicios adaptados al marco nacional de países según capacidad y de manera progresiva para el desarrollo de respuestas institucionales en cada país (organización de la oferta de CP).

Adaptando los sistemas de salud a los retos asociados con el manejo adecuado de las necesidades de los PAM con enfermedad (antes y durante el estado terminal)

La atención primaria de salud (APS) es una estrategia comprobada para maximizar los sistemas de salud 
mundiales (11). Es también una estrategia para el mejoramiento de la equidad que puede adaptarse a distintos niveles de desarrollo y diferentes contextos políticos, sociales y culturales. Además, bajo el enfoque de curso de vida, la APS se presenta como un escenario fundamental para implementar acciones desde etapas tempranas de la vida y también cuando ya se es una persona mayor, que determinen un envejecimiento saludable y activo (12). Esto es válido también para la implementación de los cuidados paliativos (13). Tener presente que la medicina o enfermería comunitaria podría ser un pilar a desarrollar, con normas o leyes que faciliten su operacionalización o ejecución de macropolíticas hacia micropolíticas a nivel local para dar cobertura de CP en los PAM(14).

Los sistemas de salud van a tener cada vez más que enfrentar a un usuario diferente: más añoso, con una fisiología, presentación clínica y patologías particulares, que pasa más tiempo enfermo, con comorbilidades (la mayoría crónicas), polimedicado, con gran potencial de discapacitarse ante la aparición o reagudización de un problema de salud; y con mayores necesidades de servicios de rehabilitación y cuidados paliativos, con una demanda cada vez incrementa de usuarios que solicitan atención ambulatoria y que no necesariamente deben hospitalizarse, ello permitiría desarrollar la estrategia de construcciones de hospitales de día para CP en América Latina (15).

Debe considerarse que con frecuencia, los problemas de salud de los PAM van más allá de ellos mismos, y condicionan una serie de situaciones y trastornos al interior de la familia, cuyo impacto rebasa el sector salud. El trabajo intersectorial es fundamental en la salud pública y el envejecimiento y es factor para el éxito en la mejora de los CP en ALC.

Cabe resaltar que para que los $\mathrm{CP}$ sean eficaces es preciso aplicar un enfoque multidisciplinario amplio que incluya a la familia y recurra a los recursos disponibles en la comunidad; pueden ponerse en práctica con eficacia incluso si los recursos son limitados, y dispensarse en centros de atención terciaria, en centros de salud comunitarios e incluso en el propio hogar. Esto requiere acciones coordinadas a nivel nacional, regional y local (16).

En ALC hay 922 servicios de CP, dirigidos a población general, lo que significa 1.63 servicios/unidades/ equipos de CP por 1000000 de habitantes. Cabe señalar que muchos de los servicios reportados no cuentan con un médico especialista en $\mathrm{CP}$ y menos con un geriatra que sirvan de consultores. El $46 \%$ (Chile y Argentina) de los servicios de CP existente en la región asisten al $10 \%$ de la población. El tipo de servicios más frecuente son equipos domiciliarios (0.4 / mil de hab). Quinientos veintitrés servicios/equipos $(0.93 / \mathrm{mil}$ de hab) actúan en el primer nivel de atención socio-sanitaria. En el segundo y tercer nivel de atención hay 1.04 servicios / mil hab y la mayoría de ellos están en hospitales de tercer nivel (2).

Ante esta situación los retos fundamentales para la optimización del escenario para la implementación de una política pública serían:

1. Establecer servicios de calidad para los PAM con enfermedad terminal en el proceso de fortalecimiento de los sistemas de salud basados en la atención primaria. Esto incluye necesariamente los mecanismos que velen por el acceso a medicamentos (incluyendo oipiodes) y el desarrollo herramientas de gestión para esto (17).

La meta debe elaborarse en función a que en un tiempo determinado, un porcentaje los países de la región contarán con una estrategia destinada a brindar CP con calidad en PAM en el marco de un servicio de salud liderado por APS.

2. Fortalecer la prevención y el manejo de enfermedades con potencial de llegar a estado terminal y otros problemas de salud asociados. Esto implica:

- Incluir las particularidades y necesidades de las personas con enfermedad terminal en los diseños de los programas y servicios para el manejo de las enfermedades crónicas y otras.

- Diseñar, adaptar y llevar a la práctica instrumentos fundamentados en pruebas científicas para elaborar programas integrales y protocolos de prevención y manejo de enfermedades crónicas con potencial de llegar a estado terminal y sobre todo las que ya estén en esta etapa (18).

- Adaptar y usar protocolos para el examen periódico de la salud del paciente con riesgo de terminalidad o con enfermedad terminal y la detección oportuna de riesgos y daños, así como para optimizar el cuidado.

Por lo tanto, la meta se definiría en función a que en un plazo determinado, al menos un porcentaje 
de los programas de prevención y manejo de las enfermedades crónicas deben abordar temas específicos sobre CP adecuados a PAM. Tener en cuenta la inequidad y el tiempo que demora en implementar una política a nivel nacional (se puede fortalecer cuidados paliativos manera centralista), o puede ser con equidad vertical o equidad horizontal.

Capacitación de los recursos humanos necesarios para atender las necesidades de cuidados paliativos de la población

Los sistemas de salud deben afrontar el reto de la atención de un usuario con características y necesidades diferentes a las habituales. Para ello, los trabajadores del sector necesitan nuevas competencias que les permitan responder a las necesidades de atención de este grupo de población (19).

Los temas relacionados con la orientación de las políticas, su institucionalización, la regulación de la educación, la calidad profesional, la calidad del trabajo, la acreditación de la educación, la certificación, el perfil del profesional y el tipo de profesional requerido y el fortalecimiento de su desempeño, son algunos de los temas sobre los que tenemos necesidad de insumos para garantizar el acceso a los cuidados paliativos. Especialmente para enfrentar barreras como la escasez de formación de los profesionales, la ausencia de un plan estructurado para su implantación y las dificultades de acceso a medicamentos (8).

Solo en 4 países de ALC los CP tienen acreditación oficial como especialidad y/o subespecialidad médica. La acreditación y los pocos programas de capacitación en el tema son muy heterogéneos. Se han estimado alrededor de 600 médicos con algún tipo de acreditación en la región (3).

En este escenario y según el ritmo de formación de los especialistas en CP va a ser muy difícil cubrir la demanda. Por lo tanto, los médicos y otros profesionales de la salud deben tener competencias que le permitan hacer un manejo primario de los CP y referir a otro nivel cuando sea necesario.

En ese sentido los retos fundamentales en relación a los actores esenciales del cambio serían:

1. Adquisición de competencias por el personal en relación con la prestación de servicios de salud de las PAM en estado terminal. Esto se debe trabajar a nivel de pregrado, posgrado y formación continua e incluyendo a los gerentes, administradores de salud y trabajadores sociales en el proceso.

La meta podría definirse en función que en un plazo determinado, todos los países de la región habrán ejecutado al menos un programa de capacitación de los trabajadores de salud en temas asociados con el cuidado paliativo en las PAM.

2. Capacitar a otros actores relacionados con la salud de las PAM (promotores comunitarios, municipios, servicios sociales, iglesias, etc.).

La meta se definiría en que en un plazo determinado, al menos $75 \%$ de los países de la región contarán con un programa de capacitación en CP destinado a las PAM con enfermedad terminal, sus cuidadores y la comunidad en general.

Perfeccionar la capacidad de generar la información necesaria para la ejecución y la evaluación de las actividades que mejoren el cuidado de la población con enfermedad terminal

Los CP exigen eficiencia, eficacia y calidad en los sistemas y servicios de salud. Para abordar esto es necesario que las instituciones académicas y de investigación asuman un papel activo en la producción de nuevos conocimientos y pruebas científicas para fundamentar la toma de decisiones relacionadas con los CP en las PAM con enfermedad terminal. Los mecanismos de información de las organizaciones adecuados y sus productos permitirán el seguimiento, la evaluación y la supervisión, así como la adaptación de los planes y estrategias.

Gracias a los sistemas de información se ha podido demostrar que el incremento al acceso a programas de CP puede resultar en ahorros financieros para los sistemas de atención de la salud (20).

En esta línea es importante cumplir los siguientes puntos:

1. Fortalecer la capacidad técnica de la autoridad sanitaria para el seguimiento y la evaluación de la atención paliativa en salud.

Por lo tanto la meta se definiría en que en un lapso determinado de tiempo, al menos un porcentaje determinado de los países usarán un sistema de 
vigilancia y evaluación de los CP en las PAM con enfermedad terminal.

2. Impulsar la obtención y difusión de las pruebas científicas necesarias para adaptar las intervenciones de salud a las realidades nacionales.

La meta se definiría en que en un determinado espacio de tiempo, al menos un porcentaje de los países habrán realizado por lo menos un estudio de investigación a nivel nacional sobre la atención paliativa en PAM con enfermedad terminal.

\section{REFERENCIAS BIBLIOGRÁFICAS}

1. OMS: el $90 \%$ de pacientes de paliativos no los recibe. Diario Médico. 2014 Enero 29: p. 2.

2. Organización Panamericana de la Salud. Taller Subregional sobre Cuidados Paliativos para Centroamérica y la República Dominicana. 2011 Noviembre 21 y 22.

3. Pastrana T, De Lima L, Wenk R, Eisenchlas J, Monti C, Rocafort $\mathrm{J}$, et al. Atlas de Cuidados Paliativos de Latinoamérica. 1st ed. ALCP, editor. Houston: IAHPC Press; 2012.

4. World Population Ageing 1950-2050. New York: United Nations, UN; 2002 United Nations. World population ageing.

5. http://www.cdc.gov/aging/pdf/ saha_2007.pdf. [Online].; 2007 [cited 201503 29. Available from: http: / /www.cdc.gov/ aging/pdf/saha_2007.pdf.

6. Cano C, Gutiérrez L, Marín P, Morales F, Peláez M, Rodríguez L, et al. Propuesta de contenidos mínimos para los programas docentes de pregrado en Medicina Geriátrica en América Latina. Rev Panam Salud Public. 2005; 17(5/6).

7. WHO. The World Health Report 2002: reducing the risks, promoting healthy life. Geneva: World Health Organization; 2002..

8. MINSA, ALMA, OPS/OMS, MIAH, SEMEG. I Foro Nacional sobre Enseñanza de Geriatría y Gerontología en el Perú. Red Latinoamericana de Gerontología. 2005;: p. 1-30.

9. Palloni A GPPM. Demographic and health conditions of ageing in Latin America and the Caribbean. Int J Epidemiol. 2002;(31): p. 762-71.

10. Pastrana T, De Lima L. Is SWOT Analysis a Useful Tool at Regional Levels? A Look at the Development of Palliative Care in Latin America (LA). In Medicine P, editor. ; 2014. p. 846.

11. Kickbusch I, Buse K. Global influences and global responses: international health at the turn of the Twenty-First Century. In Merson M, Black R, Mills A. International Public Health: Disease, Programs, Systems, and Policies. Gaithersburg, Md: Aspen Publications; 2001.

12. Parodi J, Varela L, Salinas R, Sandoval C, Alvarez L, Montenegro M. Mejorando la Salud y Reduciendo la Discapacidad en las Personas Adultas Mayores: una nueva estrategia de capacitación en medicina del Adulto Mayor para Medicos de Atención Primaria. In.; 2007.

13. Committee on the Future Health Care Workforce for Older Americans. Retooling for an aging America: Building the health care workforce. Washington D.C.:; 2008.

14. Támara LM. Estudio de cohorte descriptivo de los pacientes terminales atendidos médicamente en su domicilio en Bogotá. 2008-2012. Revista Colombiana de Anestesiología. 20 14; 42(2): p. 100-106.

15. World Health Organization. Preventing chronic diseases: a vital investment. [Online]. [cited 201208 10. Available from: http://www.who.int/chp/chronic_disease_report/part1/en /index11.html.

16. World Health Organization. Palliative care for older people: Better Practices. Copenhagen:; 2011.

17. Stjernswa“ rd J, Foley K, Ferris F. The Public Health Strategy for Palliative Care. Journal of Pain and Symptom Management. 2007; 33(5): p. 486-493.

18. Van der Steen J, Radbruch L, Hertogh C, de Boer M, Hughes J, Larkin $\mathrm{P}$, et al. White paper defining optimal palliative care in older people with dementia: A Delphi study and recommendations from the European Association for Palliative Care. Palliative Medicine. 2013;: p. 1-13.

19. Organización Panamericana de la Salud - Organización Mundial de la Salud. Plan de acción sobre la salud de las personas mayores incluido el envejecimiento activo $y$ saludable. Washington D. C. :; 2009.

20. World Health Organization. Cancer pain relief and palliative care. Report of a WHO expert committee. Technical report series, No. 804 Geneva: WHO Marketing \& Dissemination ; 1990.

\section{Fuentes de Financiamiento}

Este artículo ha sido financiado por los autores.

\section{Conflicto de interés}

Los autores declaran no tener ningún conflicto de interés

\section{Correspondencia:}

Jose F. Parodi García

Dirección: Av. La Alameda del Corregidor cdra. 15 Las Viñas - La Molina, Lima 12 - PERÚ

Teléfono: 998139858

Correo electrónico: jparodig@usmp.pe

Recibido: 08 de enero de 2016 Aprobado: 12 de febrero de 2016 